\title{
Early Paradoxical Increase of Dopamine: A Neurochemical Study of Olfactory Bulb in Asymptomatic and Symptomatic MPTP Treated Monkeys
}

\author{
Christian Pifl ${ }^{1}$, Harald Reither ${ }^{1}$, Natalia Lopez-Gonzalez del Rey ${ }^{2,3}$, Carmen Cavada ${ }^{4}$, \\ Jose A. Obeso ${ }^{2,3}$ and Javier Blesa ${ }^{2,3 *}$
}

${ }^{1}$ Center for Brain Research, Medical University of Vienna, Vienna, Austria, ${ }^{2}$ HM CINAC, Hospital Universitario HM Puerta del Sur, Mostoles, Spain, ${ }^{3}$ Centro de Investigacion Biomedica en Red sobre Enfermedades Neurodegenerativas (CIBERNED), Instituto Carlos III, Ministerio de Ciencia e Innovacion, Madrid, Spain, ${ }^{4}$ Departamento de Anatomia, Histologia y Neurociencia, Facultad de Medicina, Universidad Autonoma de Madrid, Madrid, Spain

\section{OPEN ACCESS}

Edited by: Carla Mucignat, University of Padua, Italy

Reviewed by: Inaki-Carril Mundinano, Monash University, Australia Miguel Angel García-Cabezas, Boston University, United States Elisa Garbayo,

Universidad de Navarra, Spain

*Correspondence: Javier Blesa

jblesa.hmcinac@hmhospitales.com

Received: 26 March 2017 Accepted: 12 May 2017

Published: 29 May 2017

Citation:

Pifl C, Reither H, del Rey NL, Cavada C, Obeso JA and Blesa J (2017) Early Paradoxical Increase of Dopamine: A Neurochemical Study of Olfactory Bulb in Asymptomatic and Symptomatic MPTP Treated Monkeys. Front. Neuroanat. 11:46. doi: 10.3389/fnana.2017.00046
Parkinson's disease (PD) is a neurodegenerative disease with both motor and non-motor manifestations. Hyposmia is one of the early non-motor symptoms, which can precede motor symptoms by several years. The relationship between hyposmia and $\mathrm{PD}$ remains elusive. Olfactory bulb $(\mathrm{OB})$ pathology shows an increased number of olfactory dopaminergic cells, protein aggregates and dysfunction of neurotransmitter systems. In this study we examined tissue levels of dopamine (DA) and serotonin (5-hydroxytryptamine, 5-HT) and their metabolites, of noradrenaline (NA) and of the amino acid neurotransmitters aspartate, glutamate, taurine and $\gamma$-aminobutyric acid in OBs of 1-methyl-4-phenyl-1,2,3,6-tetrahydropyridine (MPTP) treated Macaca fascicularis in different stages, including monkeys who were always asymptomatic, monkeys who recovered from mild parkinsonian signs, and monkeys with stable moderate or severe parkinsonism. DA was increased compared to controls, while neither NA and 5-HT nor the amino acid neurotransmitters were significantly changed. Furthermore, DA increased before stable motor deficits appear with $+51 \%$ in asymptomatic and $+96 \%$ in recovered monkeys. Unchanged DA metabolites suggest a special metabolic profile of the newly formed DA neurons. Significant correlation of homovanillic acid (HVA) with taurine single values within the four MPTP groups and of aspartate with taurine within the asymptomatic and recovered MPTP groups, but not within the controls suggest interactions in the OB between taurine and the DA system and taurine and the excitatory neurotransmitter triggered by MPTP. This first investigation of $\mathrm{OB}$ in various stages after MPTP administration suggests that the DA increase seems to be an early phenomenon, not requiring profound nigrostriatal neurodegeneration or PD symptoms.

Keywords: olfactory bulb, MPTP, dopamine, noradrenaline, serotonin, amino acid neurotransmitters

\section{INTRODUCTION}

The olfactory bulb $(\mathrm{OB})$ is a neuronal structure of the forebrain being the most rostral part of the adult brain in rodents, but situated on the inferior side of the forebrain in primates. According to the prosomeric model of the brain holding that the brain is formed by an uninterrupted series of transverse subunits of the neural tube, it is dorsal of the prospective anterior commissure which is 
the most rostral point of the neural tube (Puelles and Rubenstein, 2015). It functions as a processor of odor information by forming a sophisticated network of multiple types of neurons transmitting the information to the olfactory cortex. The monoamine neurotransmitters dopamine (DA), noradrenaline (NA) and serotonin (5-hydroxytryptamine, 5-HT) have been shown to influence odor processing. DA is the neurotransmitter of an intrinsic subpopulation of neurons within the glomerular cell layer and affects odor discrimination learning in rats (Escanilla et al., 2009). Recently, a direct axonal dopaminergic projection from the substantia nigra ( $\mathrm{SN}$ ) to the core of the OB was described and suggested to facilitate the perception of odorants (Höglinger et al., 2015). Noradrenergic fibers from the locus coeruleus and serotoninergic fibers from the raphe nucleus project to all layers of the OB (McLean and Shipley, 1987, 1991; Gómez et al., 2005). NA modulates odor habituation (Guerin et al., 2008) and 5-HT regulates odor inputs in the OB (Petzold et al., 2009). For all monoamines effects on synaptic transmission in the OB were also shown (Jiang et al., 1996; Berkowicz and Trombley, 2000; Petzold et al., 2009).

Importantly, olfactory dysfunction is a frequent non-motor symptom of Parkinson's disease (PD). There is structural pathology observed in the $\mathrm{OB}$ of individuals with PD. Lewy body pathology was also detected in the $\mathrm{OB}$ in patients with incidental Lewy body disease and PD (Attems et al., 2014; Saito et al., 2016). The OB volume was found reduced in several studies (Wang et al., 2011; Brodoehl et al., 2012; Li et al., 2016), whereas the number of DA neurons was increased in some reports (Huisman et al., 2004, 2008; Mundiñano et al., 2011). Dopaminergic neurogenesis also increased in OB following i.p. 1-methyl-4-phenyl-1,2,3,6-tetrahydropyridine (MPTP) intoxication in mice (Yamada et al., 2004) and after nigrostriatal DA depletion by 6-hydroxydopamine injection in the $\mathrm{SN}$ in mice and in the medial forebrain bundle in rat (Winner et al., 2006; Chiu et al., 2014; Höglinger et al., 2015). In addition, in the $\alpha$-synuclein A53T transgenic mice there was a decrease of cholinergic neurons and acetylcholinesterase activity in mitral cell layer and an increase of dopaminergic neurons and tyrosine hydroxylase protein in the glomerular layer at 10 months (Zhang et al., 2015). Tyrosine hydroxylase expression was also found increased in the OBs of transgenic rats with $\alpha$-synuclein mutations (Lelan et al., 2011). Thus, nigrostriatal DA depletion through different approaches appears to increase dopaminergic neurogenesis in the OB. The increased number of DA neurons could be a compensatory change for the lack of inhibitory modulation of mitral cells by cholinergic, noradrenergic and serotonergic olfactory afferents which are often reduced in neurodegenerative diseases (Mundiñano et al., 2011). Similarly, increased dopaminergic tone in the $\mathrm{OB}$ of PD patients could reflect a compensatory mechanism created by the early degeneration of other neurotransmitter systems and might contribute to the olfactory dysfunction exhibited by patients with neurodegenerative disorders (Bédard et al., 2002; Winner et al., 2006; Mundiñano et al., 2011).

In MPTP parkinsonian monkeys the number of olfactory dopaminergic neurons was reported to be nearly doubled as compared to controls (Belzunegui et al., 2007) and, in fact, cholinergic $\mathrm{OB}$ innervation was decreased in this model as well as in patients with PD (Mundiñano et al., 2013). Here, we have examined the OBs of Macaca fascicularis monkeys in four motor states after progressive parkinsonism induced by repeated administration of small doses of MPTP corresponding to different degrees of nigro-striatal lesion and DA depletion (Blesa et al., 2012).

\section{MATERIALS AND METHODS}

\section{Animals}

Experiments were performed using OBs from 37 male cynomolgus monkeys (Macaca fascicularis, RC Hartelust, Tilburg, Netherlands, mean age $5.2 \pm 0.9$ years, mean weight $3.6 \pm 0.65 \mathrm{~kg})$. Animals were housed in primate cages, singly or in pairs, under controlled conditions of air exchange (16 l/min), humidity (50\%) and light/dark cycles ( 8 am to $8 \mathrm{pm}$ ); food and water were available ad libitum. All procedures were performed according to the European Council Directive 86/609/EEC and in accordance with the Society for Neuroscience Policy on the Use of Animals in Neuroscience Research. The Ethics Committee for Animal Testing of the University of Navarra approved the experimental design.

\section{Experimental Protocol}

Fourteen of the 37 monkeys were used as controls. The remaining 23 monkeys were treated with MPTP hydrochloride (Sigma, St Louis, MO, USA) using a low-dose regimen $(0.5 \mathrm{mg} / \mathrm{kg}$ in saline administered intravenously once every 2 weeks) to obtain partial, slowly progressing degeneration of the dopaminergic nigro-striatal system (Blesa et al., 2010, 2012). The number of doses for each animal ranged from 1 to 16 , and the total dose from $0.5 \mathrm{mg} / \mathrm{kg}$ to $8 \mathrm{mg} / \mathrm{kg}$. Such a wide range in number and total dose of MPTP was determined by individual susceptibility to the toxin, which varies considerably when a slow intoxication protocol is employed. This in turn allows for different degrees of motor deficit and nigro-striatal lesion. Motor performance was tested using the Kurlan Scale (Blesa et al., 2012). To satisfy the classification criteria, each animal had to remain stable for at least 1 month in the corresponding motor state before being classified in a given group (Taylor et al., 1997; Blesa et al., 2012). The striatal biochemical characterization of these animals was published before (Blesa et al., 2012).

\section{Neurochemical Analysis}

Samples were ultrasonicated in 30 volumes of perchloric acid, sodium bisulfite and 3,4-dihydroxybenzylamine as internal standard (final concentration $0.1 \mathrm{M}, 0.4 \mathrm{mM}$ and $20 \mu \mathrm{g} / \mathrm{l}$, respectively) and centrifuged at $16,100 \times g$ for $10 \mathrm{~min}$. For determination of 3,4-dihydroxyphenylacetic acid (DOPAC), 5-HT, 5-hydroxyindolacetic acid (5-HIAA) and homovanillic acid (HVA) $100 \mu \mathrm{l}$ of supernatant was injected directly into a HPLC system equipped with a LiChroCART ${ }^{\circledR}$ 250-4, RP18 , $5 \mu \mathrm{m}$ (VWR) column and 
a BAS (Bioanalytical System) electrochemical detector. The mobile phase consisted of $0.1 \mathrm{M}$ sodium acetate buffer, $\mathrm{pH}$ 4.3, containing $1 \mathrm{mM}$ EDTA, $0.2 \mathrm{mM}$ l-heptane sulfonic acid, $0.1 \%$ triethylamine, $0.2 \%$ tetrahydrofurane and $10 \%$ methanol. For determination of DA and NA, aliquots of the supernatant were extracted with alumina oxide and injected into a HPLC system as described previously (Pifl et al., 1991) with minor modifications (LiChroCART ${ }^{\circledR}$ 250-4, RP18 $\mu, 5 \mu \mathrm{m}$ column, HP Programmable Electrochemical Detector 1049A, mobile phase with $9 \%$ methanol). For determination of $\gamma$-aminobutyric acid (GABA), aspartate, glutamate, taurine and glycine the supernatant was diluted with $\mathrm{H}_{2} \mathrm{O}$ (final dilution 1:2000). One-hundred micro liter were mixed with $50 \mu \mathrm{l}$ of OPA-reagent (25 mg o-phthalaldehyde dissolved in a mixture of $0.5 \mathrm{ml}$ methanol, $25 \mu l$ 1.meercaptoethanol and $4.5 \mathrm{ml} 0.4$ sodium borate, $\mathrm{pH}$ 11) and after a reaction time of $2 \mathrm{~min}, 20 \mu \mathrm{l}$ were injected by an autosampler (AS950, Jasco). The HPLC system consisted of a RP-18 column (Chromolith-Peformance RP$18 \mathrm{e}, 100 \times 4.6 \mathrm{~mm}$, Merck) in a column-heater set at $25^{\circ} \mathrm{C}$ (BFO-04 f1, W.O. Electronics) and an aqueous mobile phase containing $0.1 \mathrm{M}$ sodium phosphate buffer, $\mathrm{pH} 6.0,0.13 \mathrm{mM}$ EDTA mixed with methanol by a high-pressure gradient system at a flow-rate of $1.2 \mathrm{ml} / \mathrm{min}$ ( 2 pumps L-7100, Merck-Hitachi) in a step-gradient of $18 \%$ methanol for $18 \mathrm{~min}$ and $38 \%$ methanol for the rest of the $25 \mathrm{~min}$ run. Derivatized amino acids were detected by a fluorescence detector (L-7480, Merck-Hitachi) with excitation at $340 \mathrm{~nm}$ and emission at $440 \mathrm{~nm}$.

\section{Statistical Analysis}

Data are presented as means \pm SEM. Differences in neurochemical parameters were analyzed by one way analysis of variance (ANOVA) followed by the Bonferroni post hoc test. Pearson correlation between the parameters were calculated for the 37 single values of all animals, the 23 single values of all MPTP monkeys, 12 single values of the asymptomatic or recovered MPTP monkeys and the 14 single values of the controls. All the analysis included in the manuscript and those included in previous related articles were blinded.

\section{RESULTS}

Monkeys were classified in four motor states at least 1 month after the last MPTP administration: the asymptomatic $(n=6)$ never displayed PD-type motor symptoms; the recovered $(n=6)$ who had motor symptoms with a maximum Kurlan scale of $11.3 \pm 1.5$, but recovered to normal behavior and were sacrificed $15.3 \pm 0.4$ weeks after recovery; the mildly parkinsonian $(n=6)$ displayed motor symptoms of a Kurlan of $16 \pm 1.2$ to $12.3 \pm 0.6$; and the severely parkinsonian $(n=5)$ with Kurlan values of $20 \pm 0.7$ to $19.5 \pm 1$ before sacrifice (Blesa et al., 2012). The mean values of the $\mathrm{OB}$ weights of the 4 motor stages and the controls varied between $23 \pm 5 \mathrm{mg}$ and $28 \pm 2 \mathrm{mg}$ and did not differ significantly.

The tissue contents of the biogenic monoamines NA, DA and 5-HT in the $\mathrm{OB}$ of controls was quite similar ranging from $0.192 \mu \mathrm{g} / \mathrm{g}$ to $0.239 \mu \mathrm{g} / \mathrm{g}$ tissue weight. NA was not significantly changed as compared to controls in the various states resulting from MPTP administration with a three fold higher level in severely parkinsonian than in mildly parkinsonian monkeys (Table 1). DA levels were increased in all groups after MPTP administration (Table 1), with the highest and significant increase by $96 \%$ in the recovered group and gradually smaller, insignificant increases in the asymptomatic group (51\%) and the parkinsonian group (37\% and $43 \%$ in mildly and severely, respectively). Single values reveal an overlap between the recovered/asymptomatic and the control group, however at least half of the values were above the range of control values (Figure 1). 5-HT and its metabolite 5-HIAA were not significantly affected by MPTP treatment at any of the various states after administration (Table $\mathbf{1}$ ).

Turnover of DA and 5-HT in the OB could be estimated by molar ratios calculated from single values DOPAC/DA, HVA/DA and 5-HIAA/5-HT. Neither DOPAC nor HVA values were affected by MPTP treatment, however the molar ratio DOPAC/DA was significantly reduced in the recovered group as compared to control (Table 2), whereas the DA turnover parameter HVA/DA was unchanged after MPTP administration, as well as the parameter for 5-HT turnover 5-HIAA/5-HT.

None of the mean values of the amino acid neurotransmitters GABA, aspartate, glutamate, taurine nor glycine was affected by MPTP significantly at any of the states after administration (Table 3). Correlation analysis of the single values between the 14 parameters all over the five experimental groups resulted in a Bonferroni-corrected significant Pearson correlation coefficient between HVA and taurine, HVA and glutamate, HVA/DA and glutamate, aspartate and taurine and between aspartate and glutamate (Table 4). None of these correlations were significant if calculated for the 14 single values in the control group. However, correlation analysis in the four

TABLE 1 | Monoamine neurotransmitter parameters $\left(\mu \mathrm{g} / \mathrm{g}_{w w}\right)$ in the olfactory bulb $(\mathrm{OB})$ in various stages of 1-methyl-4-phenyl-1,2,3,6-tetrahydropyridine (MPTP) parkinsonism.

\begin{tabular}{|c|c|c|c|c|c|}
\hline Substance & Control & Asymptomatic & Recovered & Mildly parkinsonian & Severely parkinsonian \\
\hline NA & $0.192 \pm 0.026(14)$ & $0.178 \pm 0.031(6)$ & $0.157 \pm 0.045(6)$ & $0.092 \pm 0.041(6)$ & $0.291 \pm 0.028(5)^{*}$ \\
\hline DA & $0.194 \pm 0.015(14)$ & $0.294 \pm 0.044(6)$ & $0.381 \pm 0.078(6)^{\#}$ & $0.266 \pm 0.014(6)$ & $0.278 \pm 0.028$ \\
\hline DOPAC & $0.029 \pm 0.002(14)$ & $0.032 \pm 0.006(6)$ & $0.028 \pm 0.005(6)$ & $0.028 \pm 0.004(6)$ & $0.030 \pm 0.003(5)$ \\
\hline HVA & $0.306 \pm 0.035(14)$ & $0.383 \pm 0.074(6)$ & $0.347 \pm 0.057(6)$ & $0.259 \pm 0.026(6)$ & $0.365 \pm 0.050(5)$ \\
\hline 5-HT & $0.239 \pm 0.019(14)$ & $0.189 \pm 0.015(6)$ & $0.236 \pm 0.017(6)$ & $0.199 \pm 0.024(6)$ & $0.327 \pm 0.051(5)$ \\
\hline $5-\mathrm{HIAA}$ & $0.046 \pm 0.004(14)$ & $0.036 \pm 0.008(6)$ & $0.036 \pm 0.003(6)$ & $0.036 \pm 0.004(6)$ & $0.055 \pm 0.007(5)$ \\
\hline
\end{tabular}

${ }^{*} p<0.05$ vs. Mildly parkinsonian, ${ }^{*} p<0.05$ vs. Control (analysis of variance, ANOVA followed by Bonferroni t-tests). Values are given as mean \pm SEM (n). 


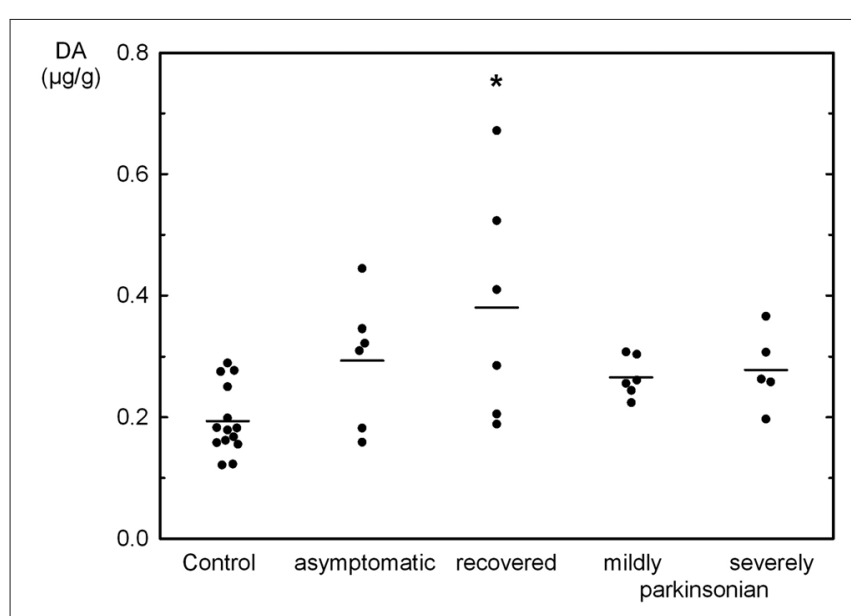

FIGURE 1 | Individual values (circles) and mean values (horizontal lines) of dopamine (DA) in the olfactory bulb (OB) of Macaca fascicularis monkeys staying after MPTP treatment either asymptomatic $(n=6)$, recovered from parkinsonian symptoms $(n=6)$, mildly $(n=6)$ or severely parkinsonian $(n=5)$ and controls $(n=14)$. ${ }^{*} P<0.05$ vs. control.

MPTP administration groups resulted still in a significant Pearson correlation coefficient between HVA and taurine, whereas correlation analysis in the two MPTP groups without parkinsonian symptoms at the time of analysis (asymptomatic and recovered group) revealed significant correlation between aspartate and taurine.

\section{DISCUSSION}

The main finding of our study is that neither the tissue content of the monoamine neurotransmitters NA, DA and 5-HT nor of their metabolites is significantly reduced at any of the various states after MPTP administration. In the same monkeys reported here striatal DA was found reduced by more than $90 \%$ (caudate in recovered group) up to $98 \%$ (caudate and putamen in the mildly and severely parkinsonian group; Blesa et al., 2012) and thalamic NA by more than 54\% (ventrolateral nucleus in the parkinsonian groups) up to $70 \%$ (ventroanterior nucleus in the severely parkinsonian group; Pifl et al., 2013). In a previous study on rhesus monkeys in a group of animals assessed asymptomatic 3-5 weeks after MPTP administration widespread loss of NA and 5-HT was observed in cortical areas with a very similar dissectional and analytical approach (Pifl et al., 1991) and some of these losses are also observed in the Macaca fascicularis used in this study (unpublished observation). This means that noradrenergic and serotoninergic innervation of the OB by locus coeruleus and raphe nucleus, respectively, react quite differently to MPTP as compared to fibers innervating thalamic and cortical brain areas.

The second novel finding of this study is that DA tissue content in $\mathrm{OB}$ is increased very early throughout all MPTP treatment groups with nearly doubling of the levels in the recovered group. The most obvious explanation is an increase of the number of intrinsic DA neurons in the $\mathrm{OB}$ reported in several studies on PD (Huisman et al., 2004; Mundiñano et al., 2011) and in animal models with nigrostriatal dopaminergic denervation by MPTP (Yamada et al., 2004; Belzunegui et al., 2007) or by 6-hydroxydopamine (Winner et al., 2006; Chiu et al., 2014). The 51\% DA increase in the asymptomatic and the robust $96 \%$ DA increment in the recovered group is in keeping with neurogenesis or formation by phenotype change of DA cells in the $O B$ in the presence of mild/moderate nigrostriatal degeneration in these monkeys (Blesa et al., 2012). Assuming that primates behave similarly to rodents, where a dopaminergic nigroolfactory projection was described recently (Höglinger et al., 2015), the small increase of DA levels in the mildly and severely parkinsonian groups might result from a combination of increased numbers of olfactory DA cells and a more profound loss of potential nigro-olfactoral nerve terminals (Figure 2). Since all nigral dopaminergic axons innervating the rat $\mathrm{OB}$ appear to branch into the striatum (Höglinger et al., 2015), the lower numbers of nigral DA cells in our parkinsonian than in the recovered monkeys (Blesa et al., 2012) might then let expect lower DA innervation of the $\mathrm{OB}$ in these groups, assuming that the nigro-olfactory projection is present in macaques as well as in rats.

The increase of dopaminergic periglomerular neurons in neurodegenerative disorders was recently explained by potentially reduced noradrenergic and serotonergic inputs with a subsequent reduced inhibitory modulation of the mitral cells resulting in hyperactivity, which might then induce an increase in the dopaminergic periglomerular neurons as a compensatory plastic response to the lack of centrifugal inhibition (Mundiñano et al., 2011). Our data of unchanged NA and 5-HT levels in the recovered group as compared to controls do not support such an explanation for increased DA neurons in the OB, however a potential cholinergic denervation, supporting centrifugal inhibition in a recent study on $\mathrm{PD}$ patients and MPTP-treated (Mundiñano et al., 2013), was not determined in our study.

Interestingly, DA increase was not accompanied by equivalent increases in the DA metabolites DOPAC and HVA, which

TABLE 2 | Dopamine (DA) and serotonin (5-hydroxytryptamine, 5-HT) turnover (molar ratio) in the OB in various stages of MPTP parkinsonism.

\begin{tabular}{|c|c|c|c|c|c|}
\hline Molar ratio & Control & Asymptomatic & Recovered & Mildly parkinsonian & Severely parkinsonian \\
\hline DOPAC/DA & $0.138 \pm 0.012(14)$ & $0.099 \pm 0.013(6)$ & $0.076 \pm 0.013(6)^{*}$ & $0.097 \pm 0.013(6)$ & $0.104 \pm 0.002(5)$ \\
\hline HVA/DA & $1.34 \pm 0.12(14)$ & $1.06 \pm 0.12(6)$ & $0.90 \pm 0.17(6)$ & $0.82 \pm 0.08(6)$ & $1.12 \pm 0.13(5)$ \\
\hline 5-HIAA/5-HT & $0.182 \pm 0.013(14)$ & $0.170 \pm 0.027(6)$ & $0.143 \pm 0.015(6)$ & $0.170 \pm 0.017(6)$ & $0.165 \pm 0.023(5)$ \\
\hline
\end{tabular}

${ }^{*} p<0.05$ vs. Control (ANOVA followed by Bonferroni t-tests). Values are given as mean \pm SEM ( $n$ ). 
TABLE 3 | Amino acid neurotransmitters $\left(\mu \mathrm{g} / \mathrm{g}_{w w}\right)$ in the OB in various stages of MPTP parkinsonism.

\begin{tabular}{|c|c|c|c|c|c|}
\hline Amino acid & Control & Asymptomatic & Recovered & Mildly parkinsonian & Severely parkinsonian \\
\hline GABA & $351 \pm 20(14)$ & $379 \pm 26(6)$ & $400 \pm 40(6)$ & $379 \pm 26(6)$ & $454 \pm 13(5)$ \\
\hline Aspartate & $230 \pm 9(14)$ & $237 \pm 18(6)$ & $231 \pm 16(6)$ & $218 \pm 11(6)$ & $232 \pm 21$ (5) \\
\hline Glutamate & $832 \pm 29(14)$ & $757 \pm 59(6)$ & $775 \pm 39(6)$ & $789 \pm 35(6)$ & $833 \pm 25(5)$ \\
\hline Taurine & $843 \pm 32(14)$ & $881 \pm 80(6)$ & $877 \pm 52(6)$ & $827 \pm 31(6)$ & $926 \pm 45(5)$ \\
\hline Glycine & $112 \pm 4$ & $99 \pm 4(6)$ & $113 \pm 13(6)$ & $103 \pm 5(6)$ & $107 \pm 6(5)$ \\
\hline
\end{tabular}

Values are given as mean $\pm \operatorname{SEM}(n)$.

TABLE 4 | Correlation between parameters with significant Pearson correlation coefficients after Bonferroni correction.

\begin{tabular}{|c|c|c|c|c|c|c|}
\hline & \multicolumn{2}{|c|}{ All five treatment groups } & \multicolumn{2}{|c|}{ All four MPTP groups } & \multicolumn{2}{|c|}{ Asymptomatic and recovered MPTP groups } \\
\hline & Correlation coefficient & $P$-value & Correlation coefficient & $P$-value & Correlation coefficient & $P$-value \\
\hline HVA and taurine & 0.663 & 0.00000765 & 0.676 & 0.000401 & - & - \\
\hline HVA and glutamate & 0.590 & 0.000121 & - & - & - & - \\
\hline HVA/DA and glutamate & 0.619 & 0.0000449 & - & - & - & - \\
\hline Aspartate and taurine & 0.551 & 0.000410 & - & - & 0.895 & 0.0000823 \\
\hline Aspartate and glutamate & 0.633 & 0.0000257 & - & - & - & - \\
\hline
\end{tabular}

explain the decrease in the turnover ratios which turned out to be significant in the case of DOPAC/DA in the recovered group. This discrepancy between a high DA increase and a lower increment of HVA and unchanged DOPAC in OB tissue with potential dopaminergic neurogenesis is surprising since the SN with its intrinsic dopaminergic neurons contains relatively higher levels of DA metabolites than striatum with its dopaminergic nerve endings but no intrinsic DA neurons (Pifl and Hornykiewicz, 2006). This would have led to expect a substantial increase of metabolites in the OB by an increased number of dopaminergic cell bodies. Therefore, a profound

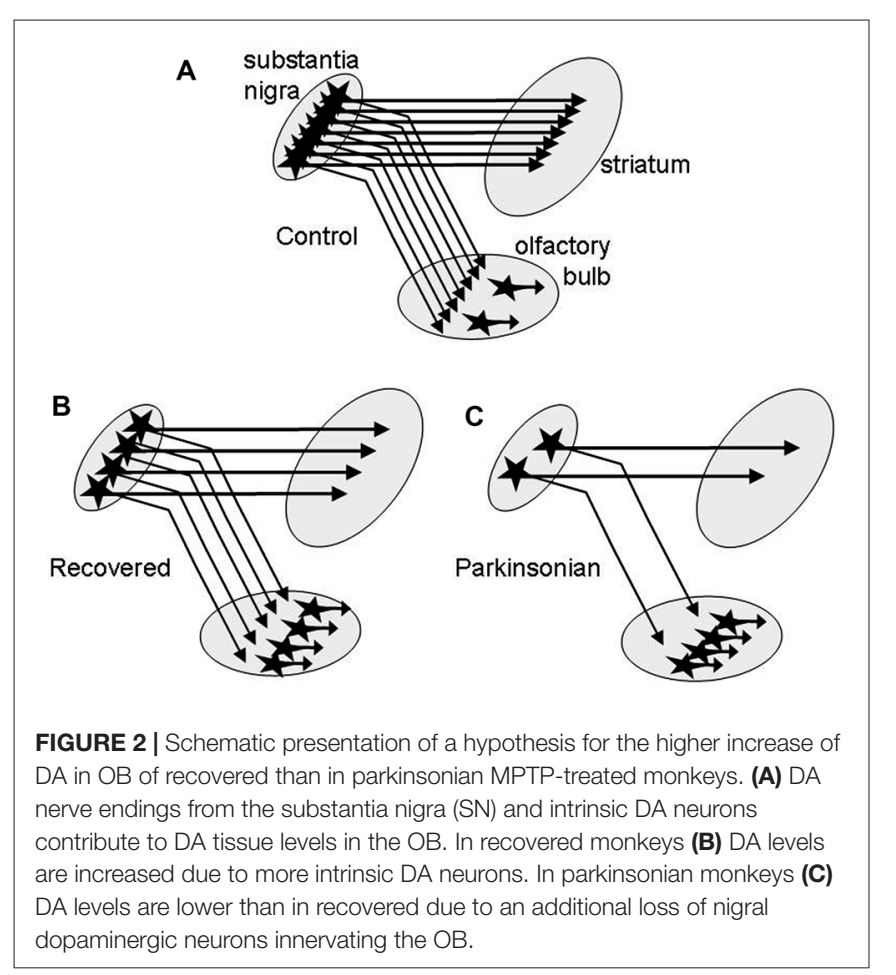

difference can be assumed between newly born dopaminergic neurons in the $\mathrm{OB}$ and dopaminergic neurons in the $\mathrm{SN}$ in terms of metabolism. For example, synaptic vesicles with vesicular monoamine transporter within newly formed dopaminergic neurons in the $\mathrm{OB}$ as opposed to the endogenous without it (Peter et al., 1995; Weihe et al., 2006) might explain the high level of DA relative to DA metabolites by preventing cytosolic degradation and a high resistance of these cells to toxic effects of MPTP similarly to mice overexpressing the vesicular monoamine transporter which were reported to be protected from MPTP neurotoxicity (Lohr et al., 2014). Furthermore, the OB has dramatically less DA transporter compared with striatum (Mitsumoto et al., 2005; Cockerham et al., 2016) which is consistent with the resistance of these neurons (in all species) to destruction by either 6-hydroxydopamine or MPTP. In fact, intranasal MPTP administration in rat also leaves many dopaminergic periglomerular neurons survive, whereas dopaminergic neurons of the $\mathrm{SN}$ were strongly affected confirming a different vulnerability to MPTP (Prediger et al., 2009). As far as potential interference of high DA levels with normal OB function is concerned the low DA turnover based on metabolite to DA ratios could indicate a defective neurotransmission of the additional dopaminergic neurons.

Out of all the neurotransmitter related substances in $\mathrm{OB}$ analyzed in this study, taurine had the highest tissue levels which is in agreement with a report on taurine content of eight different major rat CNS areas with the highest value in the $\mathrm{OB}$ which was quite similar to the present values in monkeys (Collins, 1974). A range of cortical and subcortical regions in our Macaca fascicularis also contained much lower taurine levels than $\mathrm{OB}$ (unpublished results). The significant correlations of single values of HVA with taurine calculated over all four MPTP groups and of aspartate with taurine over the asymptomatic and the recovered but not in the control group suggest an interaction between DA metabolism and taurine as well as excitatory activity and taurine in the neuronal network of 
the OB triggered by the dopaminergic neurotoxin. MPTP does not shift the levels of these parameters to significantly changed mean values in particular groups of MPTP exposed monkeys but seems to cause perturbations with parallel individual deflections in the single animals. Although correlations do not imply causation, it is interesting in this respect that intraventricular taurine increased striatal and hypothalamic DOPAC in the rat (Panula-Lehto et al., 1992), the main DA metabolite in this species, and a neuroinhibitory effect of taurine was observed in the $\mathrm{OB}$ with selectivity for mitral and tufted cells (Belluzzi et al., 2004) which are glutamatergic cells (Nagayama et al., 2014).

\section{CONCLUSION}

Our observation of a substantial increase of DA tissue levels confirms and expands to the non-human primate a paradoxical reaction of $\mathrm{DA}$ in the $\mathrm{OB}$ in parallel with progressive nigrostriatal dopaminergic lesion. We also show that no other monoamine neurotransmitters or established and potential amino acid neurotransmitters are affected by MPTP. This first neurochemical investigation of OB in various stages after MPTP administration suggests that the effect on DA seems to be an early phenomenon in $\mathrm{PD}$, not requiring profound nigrostriatal

\section{REFERENCES}

Attems, J., Walker, L., and Jellinger, K. A. (2014). Olfactory bulb involvement in neurodegenerative diseases. Acta Neuropathol. 127, 459-475. doi: 10.1007/s00401-014-1261-7

Bédard, A., Lévesque, M., Bernier, P. J., and Parent, A. (2002). The rostral migratory stream in adult squirrel monkeys: contribution of new neurons to the olfactory tubercle and involvement of the antiapoptotic protein Bcl-2. Eur. J. Neurosci. 16, 1917-1924. doi: 10.1046/j.1460-9568.2002.02263.x

Belluzzi, O., Puopolo, M., Benedusi, M., and Kratskin, I. (2004). Selective neuroinhibitory effects of taurine in slices of rat main olfactory bulb. Neuroscience 124, 929-944. doi: 10.1016/j.neuroscience.2003.12.032

Belzunegui, S., San Sebastián, W., Garrido-Gil, P., Izal-Azcárate, A., VázquezClaverie, M., Lopez, B., et al. (2007). The number of dopaminergic cells is increased in the olfactory bulb of monkeys chronically exposed to MPTP. Synapse 61, 1006-1012. doi: 10.1002/syn.20451

Berkowicz, D. A., and Trombley, P. Q. (2000). Dopaminergic modulation at the olfactory nerve synapse. Brain Res. 855, 90-99. doi: 10.1016/s00068993(99)02342-2

Blesa, J., Juri, C., Collantes, M., Peñuelas, I., Prieto, E., Iglesias, E., et al. (2010). Progression of dopaminergic depletion in a model of MPTP-induced Parkinsonism in non-human primates. An 18F-DOPA and 11C-DTBZ PET study. Neurobiol. Dis. 38, 456-463. doi: 10.1016/j.nbd.2010.03.006

Blesa, J., Pifl, C., Sánchez-González, M. A., Juri, C., García-Cabezas, M. A., Adánez, R., et al. (2012). The nigrostriatal system in the presymptomatic and symptomatic stages in the MPTP monkey model: a PET, histological and biochemical study. Neurobiol. Dis. 48, 79-91. doi: 10.1016/j.nbd.2012.05.018

Brodoehl, S., Klingner, C., Volk, G. F., Bitter, T., Witte, O. W., and Redecker, C. (2012). Decreased olfactory bulb volume in idiopathic Parkinson's disease detected by 3.0-tesla magnetic resonance imaging. Mov. Disord. 27, 1019-1025. doi: $10.1002 / \mathrm{mds} .25087$

Chiu, W. H., Carlsson, T., Depboylu, C., Höglinger, G. U., Oertel, W. H., and Ries, V. (2014). Selegiline normalizes, while l-DOPA sustains the increased number of dopamine neurons in the olfactory bulb in a 6-OHDA mouse model of Parkinson's disease. Neuropharmacology 79, 212-221. doi: 10.1016/j. neuropharm.2013.11.014 neurodegeneration or the appearance of PD motor features. Furthermore, this study supports the accumulating evidence that neurotransmitters play a crucial role in determining survival and differentiation of newly generated neurons in the $\mathrm{OB}$.

\section{AUTHOR CONTRIBUTIONS}

$\mathrm{CP}$ and JB designed the work. $\mathrm{CP}, \mathrm{HR}, \mathrm{NLR}, \mathrm{CC}, \mathrm{JAO}$ and JB did the acquisition, analysis, or interpretation of data for the work. All the authors drafted the work and revised it critically for important intellectual content approved the final version to be published.

\section{ACKNOWLEDGMENTS}

This work was funded by grants from the Ministerio de Economía y Competitividad: SAF2015-67239-P; Instituto de Salud Carlos III (CIBERNED) SAF2016-78207, Ministerio de Ciencia e Innovación, Instituto de Salud Carlos III-Fondos FEDER, a way to build Europe FIS PIE14/00034 to JAO and by the chair UAM-Fundación Tatiana Pérez de Guzmán el Bueno to CC.

Cockerham, R., Liu, S., Cachope, R., Kiyokage, E., Cheer, J. F., Shipley, M. T., et al. (2016). Subsecond regulation of synaptically released dopamine by COMT in the olfactory bulb. J. Neurosci. 36, 7779-7785. doi: 10.1523/JNEUROSCI.065816.2016

Collins, G. G. S. (1974). The rates of synthesis, uptake, and disappearance of $\left[{ }^{14} \mathrm{C}\right]$ taurine in eight areas of the rat central nervous system. Brain Res. 76, 447-459. doi: 10.1016/0006-8993(74)90821-x

Escanilla, O., Yuhas, C., Marzan, D., and Linster, C. (2009). Dopaminergic modulation of olfactory bulb processing affects odor discrimination learning in rats. Behav. Neurosci. 123, 828-833. doi: 10.1037/a0015855

Gómez, C., Briñón, J. G., Barbado, M. V., Weruaga, E., Valero, J., and Alonso, J. R. (2005). Heterogeneous targeting of centrifugal inputs to the glomerular layer of the main olfactory bulb. J. Chem. Neuroanat. 29, 238-254. doi: 10.1016/j. jchemneu.2005.01.005

Guerin, D., Peace, S. T., Didier, A., Linster, C., and Cleland, T. A. (2008). Noradrenergic neuromodulation in the olfactory bulb modulates odor habituation and spontaneous discrimination. Behav. Neurosci. 122, 816-826. doi: $10.1037 / \mathrm{a} 0012522$

Höglinger, G. U., Alvarez-Fischer, D., Arias-Carrión, O., Djufri, M., Windolph, A., Keber, U., et al. (2015). A new dopaminergic nigro-olfactory projection. Acta Neuropathol. 130, 333-348. doi: 10.1007/s00401-015-1451-y

Huisman, E., Uylings, H. B., and Hoogland, P. V. (2004). A 100\% increase of dopaminergic cells in the olfactory bulb may explain hyposmia in Parkinson's disease. Mov. Disord. 19, 687-692. doi: 10.1002/mds.10713

Huisman, E., Uylings, H. B., and Hoogland, P. V. (2008). Gender-related changes in increase of dopaminergic neurons in the olfactory bulb of Parkinson's disease patients. Mov. Disord. 23, 1407-1413. doi: 10.1002/mds. 22009

Jiang, M., Griff, E. R., Ennis, M., Zimmer, L. A., and Shipley, M. T. (1996). Activation of locus coeruleus enhances the responses of olfactory bulb mitral cells to weak olfactory nerve input. J. Neurosci. 16, 6319-6329.

Lelan, F., Boyer, C., Thinard, R., Rémy, S., Usal, C., Tesson, L., et al. (2011). Effects of human Alpha-Synuclein A53T-A30P mutations on SVZ and local olfactory bulb cell proliferation in a transgenic rat model of Parkinson disease. Parkinsons Dis. 2011:987084. doi: 10.4061/2011/ 987084 
Li, J., Gu, C.-Z., Su, J.-B., Zhu, L.-H., Zhou, Y., Huang, H.-Y., et al. (2016). Changes in olfactory bulb volume in Parkinson's disease: a systematic review and metaanalysis. PLoS One 11:e0149286. doi: 10.1371/journal.pone.0149286

Lohr, K. M., Bernstein, A. I., Stout, K. A., Dunn, A. R., Lazo, C. R., Alter, S. P., et al. (2014). Increased vesicular monoamine transporter enhances dopamine release and opposes Parkinson disease-related neurodegeneration in vivo. Proc. Natl. Acad. Sci. U S A 111, 9977-9982. doi: 10.1073/pnas.1402134111

McLean, J. H., and Shipley, M. T. (1987). Serotonergic afferents to the rat olfactory bulb: II. Changes in fiber distribution during development. J. Neurosci. 7, 3029-3039.

McLean, J. H., and Shipley, M. T. (1991). Postnatal development of the noradrenergic projection from locus coeruleus to the olfactory bulb in the rat. J. Comp. Neurol. 304, 467-477. doi: 10.1002/cne.903040310

Mitsumoto, Y., Mori, A., Ohashi, S., Nakai, M., and Moriizumi, T. (2005). Differential effects of 1-methyl-4-phenyl-1,2,3,6-tetrahydropyridine in the olfactory bulb and the striatum in mice. Neurosci. Res. 51, 111-115. doi: 10.1016/j.neures.2004.09.012

Mundiñano, I.-C., Caballero, M. C., Ordóñez, C., Hernandez, M., DiCaudo, C., Marcilla, I., et al. (2011). Increased dopaminergic cells and protein aggregates in the olfactory bulb of patients with neurodegenerative disorders. Acta Neuropathol. 122, 61-74. doi: 10.1007/s00401-011-0830-2

Mundiñano, I.-C., Hernandez, M., Dicaudo, C., Ordoñez, C., Marcilla, I., Tuñon, M.-T., et al. (2013). Reduced cholinergic olfactory centrifugal inputs in patients with neurodegenerative disorders and MPTP-treated monkeys. Acta Neuropathol. 126, 411-425. doi: 10.1007/s00401-013-1144-3

Nagayama, S., Homma, R., and Imamura, F. (2014). Neuronal organization of olfactory bulb circuits. Front. Neural Circuits 8:98. doi: 10.3389/fncir.2014. 00098

Panula-Lehto, E., Mäkinen, M., and Ahtee, L. (1992). Effects of taurine, homotaurine and GABA on hypothalamic and striatal dopamine metabolism. Naunyn Schmiedebergs Arch. Pharmacol. 346, 57-62. doi: 10.1007/bf00167571

Peter, D., Liu, Y., Sternini, C., de Giorgio, R., Brecha, N., and Edwards, R. H. (1995). Differential expression of two vesicular monoamine transporters. J. Neurosci. 15, 6179-6188.

Petzold, G. C., Hagiwara, A., and Murthy, V. N. (2009). Serotonergic modulation of odor input to the mammalian olfactory bulb. Nat. Neurosci. 12, 784-791. doi: $10.1038 / \mathrm{nn} .2335$

Pifl, C., and Hornykiewicz, O. (2006). Dopamine turnover is upregulated in the caudate/putamen of asymptomatic MPTP-treated rhesus monkeys. Neurochem. Int. 49, 519-524. doi: 10.1016/j.neuint.2006.03.013

Pifl, C., Hornykiewicz, O., Blesa, J., Adánez, R., Cavada, C., and Obeso, J. A. (2013). Reduced noradrenaline, but not dopamine and serotonin in motor thalamus of the MPTP primate: relation to severity of parkinsonism. J. Neurochem. 125, 657-662. doi: 10.1111/jnc. 12162

Pifl, C., Schingnitz, G., and Hornykiewicz, O. (1991). Effect of 1-methyl-4-phenyl1,2,3,6-tetrahydropyridine on the regional distribution of brain monoamines in the rhesus monkey. Neuroscience 44, 591-605. doi: 10.1016/03064522(91)90080-8
Prediger, R. D., Rial, D., Medeiros, R., Figueiredo, C. P., Doty, R. L., and Takahashi, R. N. (2009). Risk is in the air: an intranasal MPTP (1-methyl-4-phenyl-1,2,3,6-tetrahydropyridine) rat model of Parkinson's disease. Ann. N Y Acad. Sci. 1170, 629-636. doi: 10.1111/j.1749-6632.2009. 03885.x

Puelles, L., and Rubenstein, J. L. R. (2015). A new scenario of hypothalamic organization: rationale of new hypotheses introduced in the updated prosomeric model. Front. Neuroanat. 9:27. doi: 10.3389/fnana.2015. 00027

Saito, Y., Shioya, A., Sano, T., Sumikura, H., Murata, M., and Murayama, S. (2016). Lewy body pathology involves the olfactory cells in Parkinson's disease and related disorders. Mov. Disord. 31, 135-138. doi: 10.1002/mds.26463

Taylor, J. R., Elsworth, J. D., Roth, R. H., Sladek, J. R. Jr., and Redmond, D. E. Jr. (1997). Severe long-term 1-methyl-4-phenyl-1,2,3,6-tetrahydropyridineinduced parkinsonism in the vervet monkey (Cercopithecus aethiops sabaeus). Neuroscience 81, 745-755. doi: 10.1016/s0306-4522(97)00214-5

Wang, J., You, H., Liu, J. F., Ni, D. F., Zhang, Z. X., and Guan, J. (2011). Association of olfactory bulb volume and olfactory sulcus depth with olfactory function in patients with Parkinson disease. Am. J. Neuroradiol. 32, 677-681. doi: 10.3174/ajnr.a2350

Weihe, E., Depboylu, C., Schütz, B., Schäfer, M. K., and Eiden, L. E. (2006). Three types of tyrosine hydroxylase-positive CNS neurons distinguished by dopa decarboxylase and VMAT2 co-expression. Cell. Mol. Neurobiol. 26, 659-678. doi: 10.1007/s10571-006-9053-9

Winner, B., Geyer, M., Couillard-Despres, S., Aigner, R., Bogdahn, U., Aigner, L., et al. (2006). Striatal deafferentation increases dopaminergic neurogenesis in the adult olfactory bulb. Exp. Neurol. 197, 113-121. doi: 10.1016/j.expneurol. 2005.08.028

Yamada, M., Onodera, M., Mizuno, Y., and Mochizuki, H. (2004). Neurogenesis in olfactory bulb identified by retroviral labeling in normal and 1-methyl4-phenyl-1,2,3,6-tetrahydropyridine-treated adult mice. Neuroscience 124, 173-181. doi: 10.1016/j.neuroscience.2003.10.040

Zhang, S., Xiao, Q., and Le, W. (2015). Olfactory dysfunction and neurotransmitter disturbance in olfactory bulb of transgenic mice expressing human A53T mutant alpha-synuclein. PLoS One 10:e0119928. doi: 10.1371/journal.pone. 0119928

Conflict of Interest Statement: The authors declare that the research was conducted in the absence of any commercial or financial relationships that could be construed as a potential conflict of interest.

Copyright (c) 2017 Pifl, Reither, del Rey, Cavada, Obeso and Blesa. This is an open-access article distributed under the terms of the Creative Commons Attribution License (CC BY). The use, distribution or reproduction in other forums is permitted, provided the original author(s) or licensor are credited and that the original publication in this journal is cited, in accordance with accepted academic practice. No use, distribution or reproduction is permitted which does not comply with these terms. 